\title{
3-Point Block Methods for Direct Integration of General Second-Order Ordinary Differential Equations
}

\author{
J. O. Ehigie, ${ }^{1}$ S. A. Okunuga, ${ }^{1}$ and A. B. Sofoluwe ${ }^{2}$ \\ ${ }^{1}$ Department of Mathematics, Faculty of Science, University of Lagos, Akoka, Yaba, Lagos, Nigeria \\ ${ }^{2}$ Department of Computer Sciences, Faculty of Science, University of Lagos, Akoka, Yaba, Lagos, Nigeria
}

Correspondence should be addressed to S. A. Okunuga, nugabola@yahoo.com

Received 3 February 2011; Revised 18 April 2011; Accepted 26 May 2011

Academic Editor: István Faragó

Copyright (C) 2011 J. O. Ehigie et al. This is an open access article distributed under the Creative Commons Attribution License, which permits unrestricted use, distribution, and reproduction in any medium, provided the original work is properly cited.

\begin{abstract}
A Multistep collocation techniques is used in this paper to develop a 3-point explicit and implicit block methods, which are suitable for generating solutions of the general second-order ordinary differential equations of the form $y^{\prime \prime}=f\left(x, y, y^{\prime}\right), y\left(x_{0}\right)=a, y^{\prime}\left(x_{0}\right)=b$. The derivation of both explicit and implicit block schemes is given for the purpose of comparison of results. The Stability and Convergence of the individual methods of the block schemes are investigated, and the methods are found to be 0 -stable with good region of absolute stability. The 3-point block schemes derived are tested on standard mechanical problems, and it is shown that the implicit block methods are superior to the explicit ones in terms of accuracy.
\end{abstract}

\section{Introduction}

In recent times, the integration of Ordinary Differential Equations (ODEs) are carried out using some kind of block methods. In particular, this paper discusses the general secondorder ODEs which arise frequently in the area of science, engineering and mechanical systems and are generally written in the form,

$$
y^{\prime \prime}=f\left(x, y, y^{\prime}\right), \quad y\left(x_{0}\right)=a, \quad y^{\prime}\left(x_{0}\right)=b .
$$

This problem being second order is usually or sometimes solved by reducing the ordinary differential equation into systems of first-order ordinary differential equations. Thereafter, known numerical methods, such as Runge-Kutta methods and Linear Multistep Methods (LMMs), are used to solve them. 
Development of LMM for solving ODEs can be generated using methods such as Taylor's Series, numerical integration, and collocation method, which are restricted by an assumed order of convergence. In this paper, we will follow suite from the previous papers of Okunuga and Ehigie [1] by deriving our new method in a multistep collocation technique introduced by Onumanyi et al. [2]. Some researchers have attempted the solution of (1.1) directly using linear multistep methods without reduction to systems of first-order ordinary differential equations, they include Brown [3], Onumanyi et al. [4], Ismail et al. [5], and Ehigie et al. [6].

Block methods for solving Ordinary Differential Equations have initially been proposed by Milne [7] who used them as starting values for predictor-corrector algorithm, Rosser [8] developed Milne's method in form of implicit methods, and Shampine and Watts [9] also contributed greatly to the development and application of block methods. Fatunla [10] gave a generalization to block methods using some definition in matrix form upon which the methods derived in this paper will follow.

Hybrid methods, using collocation technique, were discussed by Ehigie et al. [6] and the continuous linear multistep scheme (CLMS) generated was used to obtain block schemes that serve as predictor-corrector schemes which were of Stormer-Cowell type. This collocation method is preferred because it is self-starting and it is convenient for easy generation of block or parallel schemes. Also the paper will consider various properties and conditions for a convergent method.

\section{Theoretical Procedure}

The procedure for the derivation of our methods in a multistep collocation technique is discussed by the methods in previous papers by Okunuga and Ehigie [1] and Ehigie et al. [6].

Consider the second-order equation

$$
y^{\prime \prime}=f\left(x, y, y^{\prime}\right), \quad y\left(x_{0}\right)=a, \quad y^{\prime}\left(x_{0}\right)=b .
$$

The numerical solution to (2.1) can be obtained using a $k$-step explicit Linear Multistep Method (LMM) of the form

$$
\sum_{j=0}^{k} \alpha_{j} y_{n+j}=h^{2} \sum_{j=0}^{k} \beta_{j} f_{n+j},
$$

where $y_{n+j} \approx y\left(x_{n}+j h\right), f_{n+j} \equiv f\left(x_{n}+j h, y\left(x_{n}+j h\right), y^{\prime}\left(x_{n}+j h\right)\right)$, and $x_{n}$ is a discrete point at node point $n$. Where, $\alpha_{j}$ and $\beta_{j}$ are parameters to be determined and usually $\beta_{k}=0$ for an explicit scheme.

Most of the problems encountered in solving the general second-order equation (2.1) is in the evaluation of the derivative term $y^{\prime}$ present in the equation. This often makes different authors to either reduce the second-order equation to system of first-order ordinary differential equations or are restricted to solve the equation of the form $y^{\prime \prime}=f(x, y)$, while $y^{\prime}$ is set to zero. However, by the introduction a of continuous scheme, this is easily taken care of. Thus if $y(x)$ is a basis polynomial of the form

$$
y(x)=\sum_{j=0}^{p} a_{j}\left(\frac{x-x_{n}}{h}\right)^{j} .
$$


To derive an $m$ point block method, where $m$ is a positive integer, we set $p=2(m-1)$ for an explicit scheme or $p=2 m+1$ for an implicit scheme, interpolating (2.3) at points $x_{n+j}$, $j=0,1,2, \ldots, k$, and collocating $y^{\prime \prime}(x)$ at points $x_{n+j}, j=0,1,2, \ldots, k$, will result to a $(p+1)$ system of equation for arbitrary $k$, Okunuga and Ehigie [1],

$$
\begin{gathered}
y\left(x_{n+j}\right)=y_{n+j}, \quad j=0,1,2, \ldots, k-1, \\
f\left(x_{n+j}\right)=f_{n+j}, \quad j=0,1,2, \ldots, k .
\end{gathered}
$$

The coefficients $a_{0}, a_{1}, a_{2}, \ldots, a_{p}$ are obtained and substituted in (2.3) to obtain the Continuous Linear Multistep Scheme (CLMS) of the form

$$
Y(x)=\sum_{j=0}^{k-1} \alpha_{j}(x) y_{n+j}+h^{2} \sum_{j=0}^{k} \beta_{j}(x) f_{n+j} .
$$

This is evaluated for at $x_{n+i}, i=0,1,2, \ldots m$ to obtain an $m$-point block method generally represented by Fatunla [10]. With the $m$-vector $Y_{m}$ and $F_{m}$ specified as,

$$
\begin{aligned}
Y_{n} & =\left[y_{n+1}, y_{n+2}, y_{n+3}, \ldots, y_{n+m}\right]^{T}, \\
Y_{n-1} & =\left[y_{n}, y_{n-1}, y_{n-2}, \ldots, y_{n-m+1}\right]^{T}, \\
F_{n-1} & =\left[f_{n}, f_{n-1}, f_{n-2}, \ldots, f_{n-m+1}\right]^{T}, \\
F_{n} & =\left[f_{n+1}, f_{n+2}, f_{n+3}, \ldots, f_{n+m}\right]^{T} .
\end{aligned}
$$

The $r$-block, m-point EBM will be generally represented as

$$
Y_{n}=\sum_{i=0}^{r} A^{(i)} Y_{n-1}+h^{2} \sum_{i=0}^{r} B^{(i)} F_{n-1}
$$

where $A^{(i)}$ and $B^{(i)}, i=0,1,2, \ldots, r$, are $m \times m$ square matrix with elements $a_{l j}^{i}, b_{l j}^{i}$ for $l, j=$ $1,2, \ldots, m$. The block scheme (2.7) is explicit if the coefficient Matrix $B^{(0)}$ is a null matrix.

\section{Derivation of Explicit Block Methods}

To derive a 1 block 3-point Explicit Block Method (EBM) that is, $m=3$, we set $p=4$. Let $y(x)$ be a basis function so that

$$
y(x)=\sum_{j=0}^{p} a_{j}\left(\frac{x-x_{n}}{h}\right)^{j}
$$


while we interpolate (3.1) at point $x=x_{n}$ and $x_{n-1}$ and collocate $y^{\prime \prime}(x)$ at $x_{n}, x_{n-1}$, and $x_{n-2}$ to obtain a system of equations

$$
\left(\begin{array}{ccccc}
1 & 0 & 0 & 0 & 0 \\
1 & -1 & 1 & -1 & 1 \\
0 & 0 & 2 & 0 & 0 \\
0 & 0 & 2 & -6 & 12 \\
0 & 0 & 2 & -12 & 48
\end{array}\right)\left(\begin{array}{c}
a_{0} \\
a_{1} \\
a_{2} \\
a_{3} \\
a_{4}
\end{array}\right)=\left(\begin{array}{c}
y_{n} \\
y_{n-1} \\
h^{2} f_{n} \\
h^{2} f_{n-1} \\
h^{2} f_{n-2}
\end{array}\right)
$$

Solving the matrix equation above, we obtain

$$
\begin{gathered}
a_{0}=y_{n} \\
a_{1}=y_{n}-y_{n-1}+\frac{7}{24} h^{2} f_{n}+\frac{1}{4} h^{2} f_{n-1}-\frac{1}{24} h^{2} f_{n-2}, \\
a_{2}=\frac{1}{2} h^{2} f_{n} \\
a_{3}=\frac{1}{4} h^{2} f_{n}-\frac{1}{3} h^{2} f_{n-1}+\frac{1}{12} h^{2} f_{n-2} \\
a_{4}=\frac{1}{24} h^{2} f_{n}-\frac{1}{12} h^{2} f_{n-1}+\frac{1}{24} h^{2} f_{n-2} .
\end{gathered}
$$

Substituting the values $a_{0}, a_{1}, a_{2}, a_{3}$, and $a_{4}$ in (3.1), we obtain the CLMS

$$
\begin{aligned}
y(x)= & \left(\frac{x-x_{n}}{h}\right) y_{n-1}-\left(\left(\frac{x-x_{n}}{h}\right)+1\right) y_{n} \\
& +h^{2}\left(\frac{7}{24}\left(\frac{x-x_{n}}{h}\right)+\frac{1}{2}\left(\frac{x-x_{n}}{h}\right)^{2}+\frac{1}{4}\left(\frac{x-x_{n}}{h}\right)^{3}+\frac{1}{24}\left(\frac{x-x_{n}}{h}\right)^{4}\right) f_{n} \\
& +h^{2}\left(\frac{1}{4}\left(\frac{x-x_{n}}{h}\right)-\frac{1}{3}\left(\frac{x-x_{n}}{h}\right)^{3}-\frac{1}{12}\left(\frac{x-x_{n}}{h}\right)^{4}\right) f_{n-1} \\
& +h^{2}\left(-\frac{1}{24}\left(\frac{x-x_{n}}{h}\right)+\frac{1}{24}\left(\frac{x-x_{n}}{h}\right)^{3}+\frac{1}{24}\left(\frac{x-x_{n}}{h}\right)^{4}\right) f_{n-2} .
\end{aligned}
$$

On evaluating (3.4) at points $x=x_{n+i}, i=1,2,3$, we obtained the convergent explicit 3-point EBM as

$$
\begin{aligned}
& y_{n+1}=-y_{n-1}+2 y_{n}+h^{2}\left(\frac{13}{12} f_{n}-\frac{1}{6} f_{n-1}+\frac{1}{12} f_{n-2}\right) \\
& y_{n+2}=-2 y_{n-1}+3 y_{n}+h^{2}\left(\frac{21}{4} f_{n}-\frac{7}{2} f_{n-1}+\frac{5}{4} f_{n-2}\right) \\
& y_{n+3}=-3 y_{n-1}+4 y_{n}+h^{2}\left(\frac{31}{2} f_{n}-15 f_{n-1}+\frac{11}{2} f_{n-2}\right) .
\end{aligned}
$$


Differentiating (3.4) and evaluating again at the same 3 discrete points of $i$, we obtain a block of first-order derivatives which can be used to determine the derivative term in the initial value problem (2.1).

$$
\begin{gathered}
y_{n+1}^{\prime}=\frac{1}{h}\left(y_{n}-y_{n-1}\right)+h\left(\frac{53}{24} f_{n}-\frac{13}{12} f_{n-1}+\frac{3}{8} f_{n-2}\right), \\
y_{n+2}^{\prime}=\frac{1}{h}\left(y_{n}-y_{n-1}\right)+h\left(\frac{53}{8} f_{n}-\frac{77}{12} f_{n-1}+\frac{55}{24} f_{n-2}\right), \\
y_{n+3}^{\prime}=\frac{1}{h}\left(y_{n}-y_{n-1}\right)+h\left(\frac{349}{24} f_{n}-\frac{71}{4} f_{n-1}+\frac{161}{24} f_{n-2}\right) .
\end{gathered}
$$

Expressing the schemes (3.5) as block using previous definition (2.7), we obtain

$$
\left(\begin{array}{lll}
1 & 0 & 0 \\
0 & 1 & 0 \\
0 & 0 & 1
\end{array}\right)\left(\begin{array}{l}
y_{n+1} \\
y_{n+2} \\
y_{n+3}
\end{array}\right)=\left(\begin{array}{lll}
0 & -1 & 2 \\
0 & -2 & 3 \\
0 & -3 & 4
\end{array}\right)\left(\begin{array}{c}
y_{n-2} \\
y_{n-1} \\
y_{n}
\end{array}\right)+h^{2}\left(\begin{array}{ccc}
\frac{1}{12} & -\frac{1}{6} & \frac{13}{12} \\
\frac{5}{4} & -\frac{7}{2} & \frac{21}{4} \\
\frac{11}{2} & -15 & \frac{31}{2}
\end{array}\right)\left(\begin{array}{c}
f_{n-2} \\
f_{n-1} \\
f_{n}
\end{array}\right) \text {. }
$$

Equation (3.7) is therefore said to be of the form (2.7). Thus (3.7) is represented notationally as $Y_{n}=A^{(1)} Y_{n-1}+h^{2} B^{(1)} F_{n-1}$.

\section{Derivation of Implicit Block Methods}

To derive a 1 block 3-point Implicit Block Method (IBM), we also define the following terms:

$$
\begin{aligned}
& Y_{n}=\left[y_{n+1}, y_{n+2}, y_{n+3}\right]^{T}, \\
& Y_{n-1}=\left[y_{n}, y_{n-1}, y_{n-2}\right]^{T}, \\
& F_{n}=\left[f_{n+1}, f_{n+2}, f_{n+3}\right]^{T}, \\
& F_{n-1}=\left[f_{n}, f_{n-1}, f_{n-2}\right]^{T} .
\end{aligned}
$$

The 3- point IBM will be generally represented as

$$
Y_{n}=A^{(1)} Y_{n-1}+h^{2}\left(B^{(1)} F_{n-1}+B^{(0)} F_{n}\right)
$$

where $A^{(1)}, B^{(0)}$, and $B^{(1)}$ are $3 \times 3$ square matrix. Let $y(x)$ be a basis function so that

$$
y(x)=\sum_{j=0}^{p} a_{j}\left(\frac{x-x_{n}}{h}\right)^{j} .
$$


Setting $p=7$ for an implicit 3-point block scheme, we will interpolate (4.3) at points $x_{n}$ and $x_{n-1}$ and collocate $y^{\prime \prime}(x)$ at 6 points $x_{n+i}, i=-2,-1,0,1,2,3$, to obtain a system of equations represented by the matrix

$$
\left(\begin{array}{cccccccc}
1 & -1 & 1 & -1 & 1 & -1 & 1 & -1 \\
1 & 0 & 0 & 0 & 0 & 0 & 0 & 0 \\
0 & 0 & 2 & -12 & 48 & -160 & 480 & -1344 \\
0 & 0 & 2 & -6 & 12 & -20 & 30 & -42 \\
0 & 0 & 2 & 0 & 0 & 0 & 0 & 0 \\
0 & 0 & 2 & 6 & 12 & 20 & 30 & 42 \\
0 & 0 & 2 & 12 & 48 & 160 & 480 & 1344 \\
0 & 0 & 2 & 18 & 108 & 540 & 2430 & 10206
\end{array}\right)\left(\begin{array}{c}
a_{0} \\
a_{1} \\
a_{2} \\
a_{3} \\
a_{4} \\
a_{5} \\
a_{6} \\
a_{7}
\end{array}\right)=\left(\begin{array}{c}
y_{n-1} \\
y_{n} \\
h^{2} f_{n-2} \\
h^{2} f_{n-1} \\
h^{2} f_{n} \\
h^{2} f_{n+1} \\
h^{2} f_{n+2} \\
h^{2} f_{n+3}
\end{array}\right) .
$$

Solving the matrix equation, we obtain

$$
\begin{aligned}
a_{0}= & y_{n}, \\
a_{1}= & y_{n}+\frac{271}{2016} h^{2} f_{n-1}+\frac{1111}{2520} h^{2} f_{n}-y_{n-1}-\frac{451}{5040} h^{2} f_{n+1}+\frac{131}{5040} h^{2} f_{n+2}-\frac{41}{5040} h^{2} f_{n-2}, \\
& -\frac{37}{10080} h^{2} f_{n+3}, \\
a_{2}= & \frac{1}{2} f_{n} \\
a_{3}= & -\frac{1}{18} h^{2} f_{n}-\frac{1}{24} h^{2} f_{n+2}+\frac{1}{120} h^{2} f_{n-2}+\frac{1}{6} h^{2} f_{n+1}-\frac{1}{12} h^{2} f_{n-1}+\frac{1}{180} h^{2} f_{n+3}, \\
a_{4}= & -\frac{5}{48} h^{2} f_{n}-\frac{1}{288} h^{2} f_{n+2}-\frac{1}{288} h^{2} f_{n-2}+\frac{1}{18} h^{2} f_{n+1}+\frac{1}{18} h^{2} f_{n-1}, \\
a_{5}= & \frac{1}{48} h^{2} f_{n}+\frac{7}{480} h^{2} f_{n+2}-\frac{1}{480} h^{2} f_{n-2}-\frac{7}{240} h^{2} f_{n+1}-\frac{1}{480} h^{2} f_{n-1}-\frac{1}{480} h^{2} f_{n+3}, \\
a_{6}= & \frac{1}{120} h^{2} f_{n}+\frac{1}{720} h^{2} f_{n+2}+\frac{1}{720} h^{2} f_{n-2}+\frac{1}{180} h^{2} f_{n+1}-\frac{1}{180} h^{2} f_{n-1}, \\
a_{7}= & -\frac{1}{504} h^{2} f_{n}-\frac{1}{1008} h^{2} f_{n+2}+\frac{1}{5040} h^{2} f_{n-2}+\frac{1}{504} h^{2} f_{n+1}-\frac{1}{1080} h^{2} f_{n-1}-\frac{1}{5040} h^{2} f_{n+3} .
\end{aligned}
$$

Substituting the $a_{i}, i=0,1, \ldots, 7$ in (4.3), we obtain the CLMS,

$$
\begin{aligned}
y(x)= & -\left(\frac{x-x_{n}}{h}\right) y_{n-1}+\left(\left(\frac{x-x_{n}}{h}\right)+1\right) y_{n} \\
& +h^{2}\left(-\frac{451}{5040}\left(\frac{x-x_{n}}{h}\right)+\frac{1}{6}\left(\frac{x-x_{n}}{h}\right)^{3}+\frac{1}{18}\left(\frac{x-x_{n}}{h}\right)^{4}-\frac{7}{240}\left(\frac{x-x_{n}}{h}\right)^{5}\right. \\
& \left.\quad-\frac{1}{180}\left(\frac{x-x_{n}}{h}\right)^{6}+\frac{1}{504}\left(\frac{x-x_{n}}{h}\right)^{7}\right) f_{n+1}
\end{aligned}
$$


Advances in Numerical Analysis

$$
\begin{aligned}
+h^{2}( & -\frac{131}{5040}\left(\frac{x-x_{n}}{h}\right)-\frac{1}{24}\left(\frac{x-x_{n}}{h}\right)^{3}-\frac{1}{288}\left(\frac{x-x_{n}}{h}\right)^{4}+\frac{7}{480}\left(\frac{x-x_{n}}{h}\right)^{5} \\
& \left.+\frac{1}{720}\left(\frac{x-x_{n}}{h}\right)^{6}-\frac{1}{1008}\left(\frac{x-x_{n}}{h}\right)^{7}\right) f_{n+2} \\
+h^{2}( & \left.-\frac{37}{10080}\left(\frac{x-x_{n}}{h}\right)+\frac{1}{180}\left(\frac{x-x_{n}}{h}\right)^{3}-\frac{1}{480}\left(\frac{x-x_{n}}{h}\right)^{5}+\frac{1}{5040}\left(\frac{x-x_{n}}{h}\right)^{7}\right) f_{n+3} \\
+h^{2}( & -\frac{41}{5040}\left(\frac{x-x_{n}}{h}\right)+\frac{1}{120}\left(\frac{x-x_{n}}{h}\right)^{3}-\frac{1}{288}\left(\frac{x-x_{n}}{h}\right)^{4}-\frac{1}{480}\left(\frac{x-x_{n}}{h}\right)^{5} \\
& \left.+\frac{1}{720}\left(\frac{x-x_{n}}{h}\right)^{6}-\frac{1}{5040}\left(\frac{x-x_{n}}{h}\right)^{7}\right) f_{n-2} \\
+h^{2}( & \frac{271}{2016}\left(\frac{x-x_{n}}{h}\right)^{3}-\frac{1}{12}\left(\frac{x-x_{n}}{h}\right)^{3}+\frac{1}{18}\left(\frac{x-x_{n}}{h}\right)^{4}-\frac{1}{480}\left(\frac{x-x_{n}}{h}\right)^{5} \\
& \left.-\frac{1}{180}\left(\frac{x-x_{n}}{h}\right)^{6}+\frac{1}{1080}\left(\frac{x-x_{n}}{h}\right)^{7}\right) f_{n-1} \\
+h^{2}( & \frac{1111}{2520}\left(\frac{x-x_{n}}{h}\right)^{6}+\frac{1}{2}\left(\frac{x-x_{n}}{h}\right)^{2}-\frac{1}{18}\left(\frac{x-x_{n}}{h}\right)^{3}-\frac{1}{48}\left(\frac{x-x_{n}}{h}\right)^{4}+\frac{1}{48}\left(\frac{x-x_{n}}{h}\right)^{5} \\
+ & \left.\frac{1}{120}\left(\frac{x-x_{n}}{h}\right)^{6}-\frac{1}{504}\left(\frac{x-x_{n}}{h}\right)^{7}\right) f_{n} .
\end{aligned}
$$

On evaluating (4.6) at points $x=x_{n+1}, x_{n+2}$ and $x_{n+3}$, we obtain the 3-point implicit block Linear Multistep methods

$$
\begin{gathered}
y_{n+1}=-y_{n-1}+2 y_{n}+h^{2}\left(\frac{1}{10} f_{n+1}-\frac{1}{240} f_{n+2}\right)+h^{2}\left(\frac{97}{120} f_{n}+\frac{1}{10} f_{n-1}-\frac{1}{240} f_{n-2}\right), \\
y_{n+2}=-2 y_{n-1}+3 y_{n}+h^{2}\left(\frac{121}{120} f_{n+1}+\frac{11}{120} f_{n+2}-\frac{1}{240} f_{n+3}\right)+h^{2}\left(\frac{103}{60} f_{n}+\frac{47}{240} f_{n-1}-\frac{1}{120} f_{n-2}\right), \\
y_{n+3}=-3 y_{n-1}+4 y_{n}+h^{2}\left(\frac{29}{15} f_{n+1}+\frac{127}{120} f_{n+2}+\frac{1}{15} f_{n+3}\right)+h^{2}\left(\frac{161}{60} f_{n}+\frac{4}{15} f_{n-1}-\frac{1}{120} f_{n-2}\right) .
\end{gathered}
$$

On differentiating (4.6) and evaluating again at the same 3 discrete points of $x$, we obtain

$$
\begin{aligned}
y_{n+1}^{\prime}= & \frac{1}{h}\left(y_{n}-y_{n-1}\right)+h\left(\frac{589}{1260} f_{n+1}-\frac{389}{10080} f_{n+2}+\frac{1}{252} f_{n+3}\right) \\
& +h\left(\frac{5029}{5040} f_{n}+\frac{22}{315} f_{n-1}-\frac{1}{2016} f_{n-2}\right),
\end{aligned}
$$




$$
\begin{aligned}
y_{n+2}^{\prime}= & \frac{1}{h}\left(y_{n}-y_{n-1}\right)+h\left(\frac{5933}{5040} f_{n+1}+\frac{407}{1008} f_{n+2}-\frac{149}{10080} f_{n+3}\right) \\
& +h\left(\frac{2063}{2520} f_{n}+\frac{1243}{10080} f_{n-1}-\frac{41}{5040} f_{n-2}\right), \\
y_{n+3}^{\prime}= & \frac{1}{h}\left(y_{n}-y_{n-1}\right)+h\left(\frac{157}{252} f_{n+1}+\frac{14059}{10080} f_{n+2}+\frac{397}{1260} f_{n+3}\right) \\
& +h\left(\frac{5813}{5040} f_{n}+\frac{1}{315} f_{n-1}+\frac{107}{10080} f_{n-2}\right) .
\end{aligned}
$$

The derivative formulae will be used to obtain the first derivative term in (2.1). Expressing the schemes (4.7) as block using our previous definition according to Fatunla [10], (4.7) becomes

$$
\begin{aligned}
\left(\begin{array}{lll}
1 & 0 & 0 \\
0 & 1 & 0 \\
0 & 0 & 1
\end{array}\right)\left(\begin{array}{l}
y_{n+1} \\
y_{n+2} \\
y_{n+3}
\end{array}\right)= & \left(\begin{array}{ccc}
0 & -1 & 2 \\
0 & -2 & 3 \\
0 & -3 & 4
\end{array}\right)\left(\begin{array}{c}
y_{n-2} \\
y_{n-1} \\
y_{n}
\end{array}\right)+h^{2}\left(\begin{array}{ccc}
\frac{1}{10} & -\frac{1}{240} & 0 \\
\frac{121}{120} & \frac{11}{120} & -\frac{1}{240} \\
\frac{29}{15} & \frac{127}{120} & \frac{1}{15}
\end{array}\right)\left(\begin{array}{c}
f_{n+1} \\
f_{n+2} \\
f_{n+3}
\end{array}\right) \\
& +h^{2}\left(\begin{array}{ccc}
-\frac{1}{240} & \frac{1}{10} & \frac{97}{120} \\
-\frac{1}{120} & \frac{47}{240} & \frac{103}{60} \\
-\frac{1}{120} & \frac{4}{15} & \frac{161}{60}
\end{array}\right)\left(\begin{array}{c}
f_{n-2} \\
f_{n-1} \\
f_{n}
\end{array}\right)
\end{aligned}
$$

This scheme is also of the form (4.2).

\section{Order, Consistency, Stability, and Convergence of the Methods}

\subsection{Order of the Methods}

The methods (3.5) and (4.7) so derived are methods belonging to the class of LMM (2.2). So, if LMM (2.2) is a method associated with a linear difference operator,

$$
€[y(x) ; h]=\sum_{j=0}^{k}\left(\alpha_{j} y(x+j h)-h^{2} \beta_{j} y^{\prime \prime}(x+j h)\right)
$$

where $y(x)$ is an arbitrary function continuously differentiable on the interval $[a, b]$. The Taylors series expansion about the point $x$,

$$
€[y(x) ; h]=c_{0} y(x)+c_{1} h y^{\prime}(x)+c_{2} h^{2} y^{\prime \prime}(x)+\cdots+c_{q} h^{q} y^{(q)}(x),
$$


where

$$
\begin{gathered}
c_{0}=\alpha_{0}+\alpha_{1}+\alpha_{2}+\cdots+\alpha_{k}, \\
c_{1}=\alpha_{1}+2 \alpha_{2}+\cdots+k \alpha_{k}, \\
c_{2}=\frac{1}{2 !}\left(\alpha_{1}+2^{2} \alpha_{2}+\cdots+k^{2} \alpha_{k}\right)-\left(\beta_{1}+\beta_{2}+\cdots+\beta_{k}\right), \\
\vdots \\
c_{q}=\frac{1}{q !}\left(\alpha_{1}+2^{q} \alpha_{2}+\cdots+k^{q} \alpha_{k}\right)-\frac{1}{(q-2) !}\left(\beta_{1}+2^{q-2} \beta_{2}+\cdots+k^{q-2} \beta_{k}\right), \\
q=3,4, \ldots
\end{gathered}
$$

Definition 5.1. The method (2.2) is said to be of order $p$ if

$$
c_{0}=c_{1}=c_{2}=\cdots=c_{p+1}=0, \quad c_{p+2} \neq 0 .
$$

$c_{p+2}$ is the error constant and $c_{p+2} h^{p+2} y^{(p+2)}\left(x_{n}\right)$ is the truncation error at point $x_{n}$.

Applying this definition to the individual methods (3.5) and (4.7) which make up the block explicit and implicit methods which is of the form (2.2), it is easily verified that each of the explicit methods (3.5) is of order $p=(3,3,3)^{T}$ with error constants $[1 / 12,4 / 3,41 / 6]^{T}$. Also applying this definitions on the implicit methods (4.7), the implicit method was of order $p=(6,6,6)^{T}$ with error constants $[-439 / 4320,-3479 / 2880,-1393 / 180]^{T}$.

Definition 5.2. A Linear Multistep Method of the form (2.2) is said to be consistent if the LMM is of order $p \geq 1$.

Since the methods derived in (3.5) and (4.7) are of order $p \geq 1$, therefore, the methods are consistent according to Definition 5.2.

\subsection{0-Stability of the Method}

From literature, it is known that stability of a linear multistep method determines the manner in which the error is propagated as the numerical computation proceeds. Hence, it would be necessary to investigate the stability properties of the newly developed methods. In this paper, the 0-stability and the Region of Absolute Stability (RAS) of the methods are discussed.

Definition 5.3. The first characteristic polynomial, $\rho(\xi)$, associated with the linear multistep method (2.2), where it is the polynomial of degree $k$ whose coefficients are $\alpha_{j}$ and the second characteristic polynomial $\sigma(\xi)$ whose coefficients are $\beta_{j}$, is defined by

$$
\begin{aligned}
& \rho(\xi)=\sum_{j=0}^{k} \alpha_{j} \xi^{j}, \\
& \sigma(\xi)=\sum_{j=0}^{k} \beta_{j} \xi^{j},
\end{aligned}
$$

where $\xi \in C, C$ is a set of complex numbers and a free variable. Stability is determined by the location of the roots of the characteristic polynomial. 
Definition 5.4. The block method of form (2.7) and (4.2) is said to be 0-stable if the roots $\xi_{j}$, $j=1(1) k$, of the first characteristic polynomial $\rho(\xi)=\operatorname{det}\left[\sum_{i=0}^{k} A_{i} \xi^{k-1}\right]=0, A_{0}=-I$, satisfy $|\xi| \leq 1$. If one of the roots is +1 , we call the roots the principal roots of $\rho(\xi)$.

Definition 5.5. The Region of Absolute Stability (RAS) of methods of (2.7) and (4.2) is the set

$$
\begin{gathered}
R=\left\{h^{2} \lambda: \text { for that } h^{2} \lambda\right. \text { where the roots of the stability polynomial are } \\
\text { of absolute less than one }\} .
\end{gathered}
$$

However, in this paper, the boundary locus method will be used to plot and view the RAS. This is obtained using the first and second characteristic polynomials as

$$
z(\theta)=\frac{\rho\left(e^{i \theta}\right)}{\sigma\left(e^{i \theta}\right)}
$$

Resolving this to real and imaginary parts and evaluating for values of $\theta \in(0,2 \pi)$ give the region of stability on a graph.

The stability property of the 3-point EBM is determined by applying the scheme (3.7) to the test problem, $y^{\prime \prime}=\lambda y$. By setting $z=\lambda h^{2}$, the block scheme (3.7) becomes

$$
\left(\begin{array}{lll}
1 & 0 & 0 \\
0 & 1 & 0 \\
0 & 0 & 1
\end{array}\right)\left(\begin{array}{l}
y_{n+1} \\
y_{n+2} \\
y_{n+3}
\end{array}\right)=\left(\begin{array}{ccc}
\frac{z}{12} & -1-\frac{z}{6} & 2+\frac{13 z}{12} \\
\frac{5 z}{4} & -2-\frac{7 z}{2} & 3+\frac{21 z}{4} \\
-\frac{11 z}{2} & -3-15 z & 4+\frac{31 z}{2}
\end{array}\right)\left(\begin{array}{c}
y_{n-2} \\
y_{n-1} \\
y_{n}
\end{array}\right)
$$

This is of the form $Y_{n}=\left(A^{(1)}+z B^{(1)}\right) Y_{n-1}$. The stability polynomial of this is given as

$$
\operatorname{det}\left(\xi-\left(A^{(1)}+z B^{(1)}\right)\right)=0
$$

Hence the stability polynomial of the 3-point EBM (3.7) is

$$
\xi^{3}+\xi^{2}\left(-\frac{145}{12} z-2\right)+\xi\left(\frac{79}{4} z^{2}+\frac{37}{6} z+1\right)+\left(-z^{3}+\frac{5}{4} z^{2}-\frac{37}{12} z\right)=0
$$

Substituting $z=0$ in (5.9), we obtain all the roots of the derived equation to be less than or equal to 1; hence it shows that the 3-point EBM generated is 0-stable.

Similarly, this is extended to the 3-point implicit block method (IBM) given in (4.9) and the stability polynomial obtained is

$$
\operatorname{det}\left(\xi\left(I-z B^{(0)}\right)-\left(A^{(1)}+z B^{(1)}\right)\right)=0
$$


which gives

$$
\begin{gathered}
\xi^{3}\left(-\frac{59}{43200} z^{3}+\frac{11}{360} z^{2}-\frac{31}{120} z+1\right)+\xi^{2}\left(-\frac{11957}{21600} z^{3}-\frac{2153}{480} z^{2}-\frac{679}{80} z-2\right) \\
+\xi\left(\frac{89}{4320} z^{3}+\frac{1}{15} z^{2}-\frac{1}{4} z+1\right)-\left(\frac{1}{21600} z^{3}-\frac{1}{1440} z^{2}+\frac{137}{240} z\right)=0
\end{gathered}
$$

Substituting $z=0$ in (5.11), we obtain all the roots of the derived equation to be less than or equal to 1; hence it shows that the 3-point IBM generated is 0-stable.

Theorem 5.6. The LMM (2.2) is convergent iff it is consistent and 0-Stable.

The proof is given in Fatunla [11] and Lambert [12].

Since the consistency and 0-stability of the methods have been established, then the explicit block method (3.5) and the implicit block method (4.7) are convergent.

The Region of Absolute Stability (RAS) of the block methods in this paper are drawn based on the third scheme of the block. The RAS of the linear multistep methods in the EBM (3.5) is drawn with the Maple software and displayed in Figure 1 below while the RAS for the implicit block method (4.7) is displayed in Figure 2.

It is observed that the RAS of the IBM is wider in range than the RAS of the EBM. This means that the implicit schemes will cope with Initial Value Problems better than the EBM in implementation with a higher step length.

\section{Implementation of Schemes Generated}

A Matlab code was developed for the implementation of the schemes in Sections 3 and 4 above. The code was designed so that it determines the initial points of the starting block methods with the analytical solution if it exists.

Thereafter it generated the values for $y_{n+1}, y_{n+2}$, and $y_{n+3}$, using the block schemes directly for the explicit schemes and predictor-corrector technique for the implicit schemes using a fixed step size $h$. So for $v=10$ corrections, the sequence of computation follows the $P(E C)^{v}$, where $P, E$, and $C$ denote Predicting, Evaluating and Correcting as it is generally used in Predictor-Corrector modes for numerical computations with a desired accuracy Lambert [12].

\section{Numerical Experiment}

\subsection{Experimental Problems}

In this paper three standard problems are considered and our newly developed methods are used to solve these problems. The problems are presented below.

Problem 1. Consider the test problem for second-order ODE given by

$$
y^{\prime \prime}=\lambda y, \quad y(0)=y^{\prime}(0)=1, \quad \text { with } \lambda=-1,0 \leq x \leq 1
$$




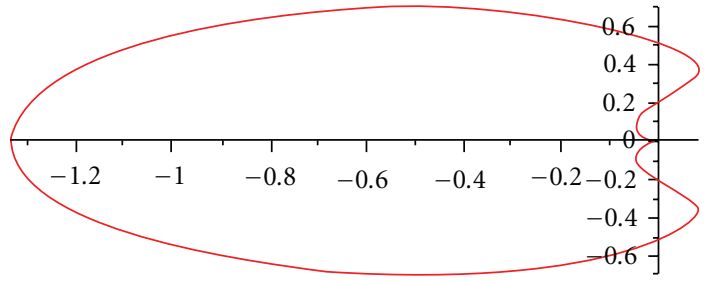

Figure 1: RAS for the explicit scheme.

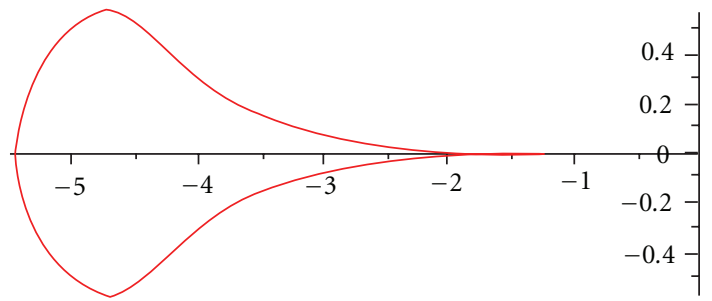

Figure 2: RAS for implicit scheme.

This problem is known to have an analytical solution of $y(x)=\cos x+\sin x$, and the results are presented in Table 1.

Problem 2. The Van der Pol equation which describes the Van der Pol oscillator is the secondorder ODE

$$
y^{\prime \prime}=\mu\left(1-y^{2}\right) y^{\prime}-\lambda y, \quad y(0)=A, \quad y^{\prime}(0)=B, \quad 0 \leq x \leq 1 .
$$

and it assumes some real positive numbers $\mu$ and $\lambda$. The problem was named after B. Van der Pol in 1926. This equation has attracted a lot of research attention both in nonlinear mechanics and control theory. This equation has no solution in terms of known tabulated transcendental function Fatunla [11]. To solve this directly using the schemes generated, we solve for $\mu=$ $10^{-4}, 10^{-6}$, and $10^{-8}$ with $\lambda=A=B=1$. However, as $\mu=0,(7.2)$ has the analytical solution

$$
y(x)=\cos (x)+\sin (x)
$$

The results are presented using Maximum Error which is given in Table 2.

Problem 3. The third problem is the second order ODE

$$
y^{\prime \prime}=-101 y^{\prime}-100 y, \quad y(0)=1, \quad y^{\prime}(0)=0, \quad 0 \leq x \leq 1,
$$

with exact solution

$$
y=\frac{1}{99}\left(100 e^{-x}-e^{-100 x}\right)
$$


Table 1: Result of the test problem (1).

\begin{tabular}{lcc}
\hline$h$ & Explicit max. error & Implicit max. error \\
\hline 0.01 & $5.12 E-07$ & $6.55 E-14$ \\
0.005 & $6.30 E-08$ & $2.12 E-13$ \\
0.0025 & $7.81 E-09$ & $1.78 E-13$ \\
0.001 & $4.95 E-10$ & $9.43 E-13$ \\
0.0005 & $5.52 E-11$ & $8.00 E-12$ \\
0.00025 & $1.15 E-11$ & $1.77 E-11$ \\
\hline
\end{tabular}

Table 2: Result of the Van der Pol problem (2).

\begin{tabular}{lcccccc}
\hline & \multicolumn{2}{c}{$\mu=10^{-4}$} & \multicolumn{2}{c}{$\mu=10^{-6}$} & \multicolumn{2}{c}{$\mu=10^{-8}$} \\
$h$ & $\begin{array}{c}\text { Explicit } \\
\text { max. error }\end{array}$ & $\begin{array}{c}\text { Implicit } \\
\text { max. error }\end{array}$ & $\begin{array}{c}\text { Explicit } \\
\text { max. error }\end{array}$ & $\begin{array}{c}\text { Implicit } \\
\text { max. error }\end{array}$ & $\begin{array}{c}\text { Explicit } \\
\text { max. error }\end{array}$ & $\begin{array}{c}\text { Implicit } \\
\text { max. error }\end{array}$ \\
\hline 0.01 & $1.15 E-05$ & $1.10 E-05$ & $6.22 E-07$ & $1.10 E-07$ & $5.13 E-07$ & $1.10 E-09$ \\
0.005 & $1.10 E-05$ & $1.10 E-05$ & $1.72 E-07$ & $1.10 E-07$ & $6.48 E-08$ & $1.10 E-09$ \\
0.0025 & $1.10 E-05$ & $1.10 E-05$ & $1.17 E-07$ & $1.10 E-07$ & $8.90 E-09$ & $1.10 E-09$ \\
0.001 & $1.10 E-05$ & $1.10 E-05$ & $1.10 E-07$ & $1.10 E-07$ & $1.59 E-09$ & $1.10 E-09$ \\
0.0005 & $1.10 E-05$ & $1.10 E-05$ & $1.10 E-07$ & $1.10 E-07$ & $1.16 E-09$ & $1.10 E-09$ \\
0.00025 & $1.10 E-05$ & $1.10 E-05$ & $1.10 E-07$ & $1.10 E-07$ & $1.07 E-09$ & $1.10 E-09$ \\
\hline
\end{tabular}

Table 3: Result of problem (3).

\begin{tabular}{lcc}
\hline$h$ & Explicit max. error & Implicit max. error \\
\hline 0.01 & Failed & $6.90 E-06$ \\
0.005 & Failed & $7.05 E-09$ \\
0.0025 & Failed & $4.34 E-10$ \\
0.001 & $3.40 E-06$ & $2.79 E-12$ \\
0.0005 & $6.17 E-07$ & $1.10 E-13$ \\
0.00025 & $9.17 E-08$ & $5.07 E-14$ \\
\hline
\end{tabular}

The results obtained by using the 3-point EBM and IBM are presented in Table 3.

\subsection{Numerical Results}

The Numerical results for the solution of the problems illustrated in the previous subsection will be presented in form of the Maximum Error.

It would be observed that in Problems 2, the explicit methods compare favourably with the implicit scheme but the accuracy of the methods increases as $\mu$ decreases. Whereas in Problems 1 and 3 the explicit block methods produce a poorer result compared to the implicit method. However, in Problem 3 the explicit method failed for a step length $h=0.01$, 0.005 , and 0.0025 , while the implicit method shows its superiority by producing results.

\section{Conclusion}

We have been able to derive some 3-point Implicit and Explicit Block Methods via collocation multistep technique. This block schemes derived in this paper have been represented in form 
of (2.7), which is a representation given by Fatunla [10]. This representation of the schemes generated as a single block methods will yield 3 points on implementation. The Order, Stability, Consistency, and Convergence of these schemes were established as stated. These derived methods were implemented on standard mechanical problems and their results were found to be sufficiently accurate for various values of step length.

\section{References}

[1] S. A. Okunuga and J. Ehigie, "A new derivation of continuous collocation multistep methods using power series as basis function," Journal of Modern Mathematics and Statistics, vol. 3, no. 2, pp. 43-50, 2009.

[2] P. Onumanyi, D. O. Awoyemi, S. N. Jator, and U. W. Sirisena, "New linear multistep methods with continuous coefficients for first order IVPs," Journal of the Nigerian Mathematical Society, vol. 13, pp. 37-51, 1994.

[3] R. L. Brown, "Some characteristics of implicit multistep multi-derivative integration formulas," SIAM Journal on Numerical Analysis, vol. 14, no. 6, pp. 982-993, 1977.

[4] P. Onumanyi, J. Fatokun, and B. O. Adejo, "Accurate numerical differentiation by continuous integrators for ordinary differential equations," Journal of the Nigerian Mathematical Society, vol. 27, pp. 69-90, 2008.

[5] F. Ismail, L. K. Yap, and O. Mohamad, "Explicit and implicit 3-point block methods for solving special second order ordinary differential equations directly," International Journal of Mathematical Analysis, vol. 3, no. 5, pp. 239-254, 2009.

[6] J. O. Ehigie, S. A. Okunuga, and A. B. Sofoluwe, "On generalized 2-step continuous linear multistep method of hybrid type for the integration of second order ordinary differential equations," Archives of Applied Science Research, vol. 2, no. 6, pp. 362-372, 2010.

[7] W. E. Milne, Numerical Solution of Differential Equations, John Wiley \& Sons, New York, NY, USA, 1953.

[8] J. B. Rosser, "A Runge-Kutta for all seasons," SIAM Review, vol. 9, pp. 417-452, 1967.

[9] L. F. Shampine and H. A. Watts, "Block implicit one-step methods," Mathematics of Computation, vol. 23, pp. 731-740, 1969.

[10] S. O. Fatunla, "Parallel methods for second order ODEs," in Monogragh- Computational Ordinary Differential Equations, S. O. Fatunla, Ed., pp. 87-99, University Press Plc, Ibadan, Nigeria, 1992.

[11] S. O. Fatunla, Numerical Methods for Fatunla Initial Value Problems in Ordinary Differential Equations, Academic Press, New York, NY, USA, 1988.

[12] J. D. Lambert, Computational Methods in Ordinary Differential Equations, John Wiley \& Sons, New York, NY, USA, 1973. 


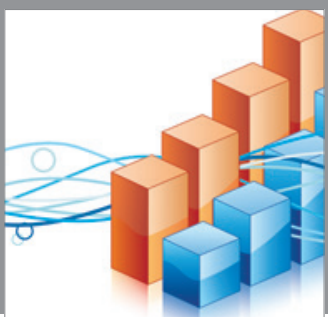

Advances in

Operations Research

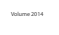

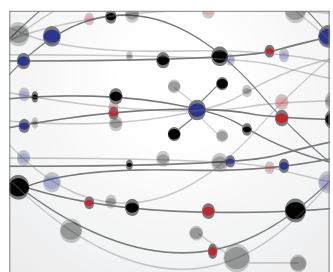

\section{The Scientific} World Journal
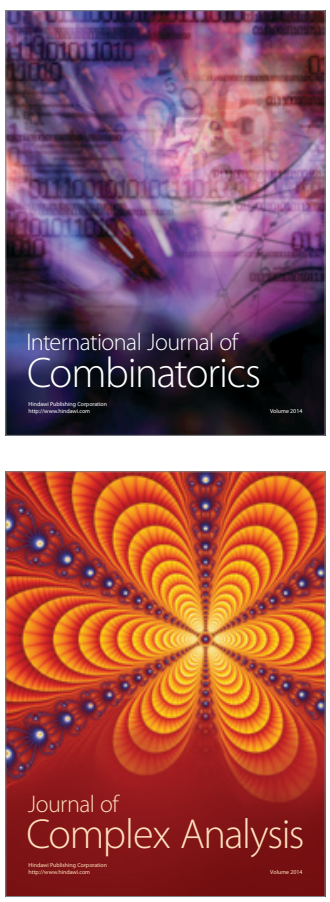

International Journal of

Mathematics and

Mathematical

Sciences
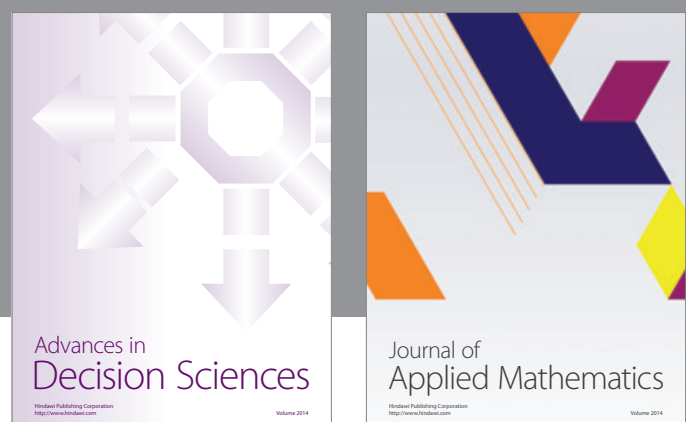

Journal of

Applied Mathematics
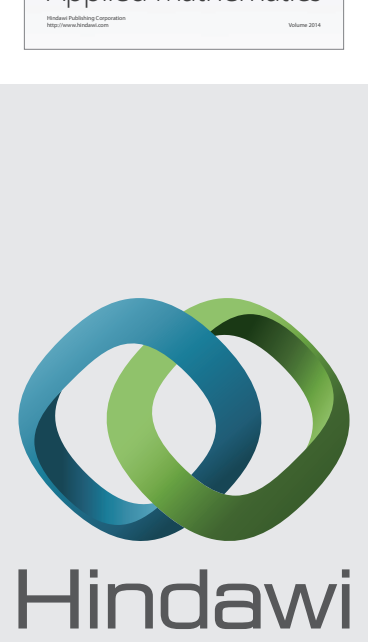

Submit your manuscripts at http://www.hindawi.com
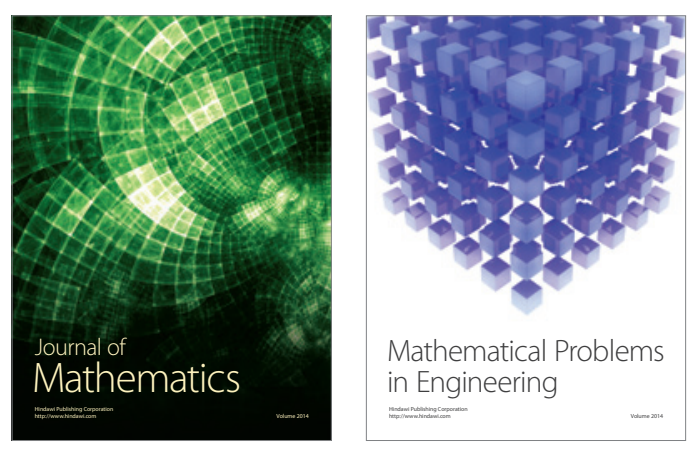

Mathematical Problems in Engineering
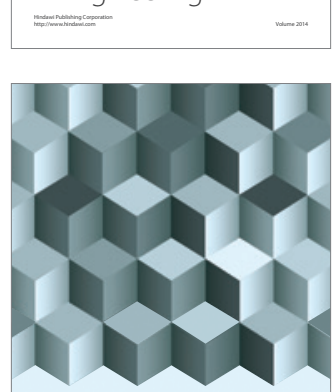

Journal of

Function Spaces
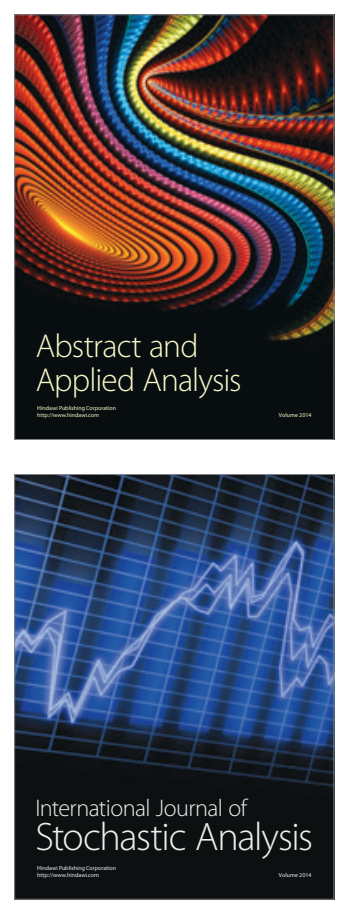

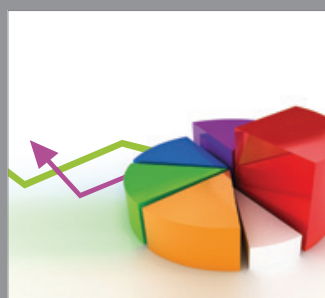

ournal of

Probability and Statistics

Promensencen
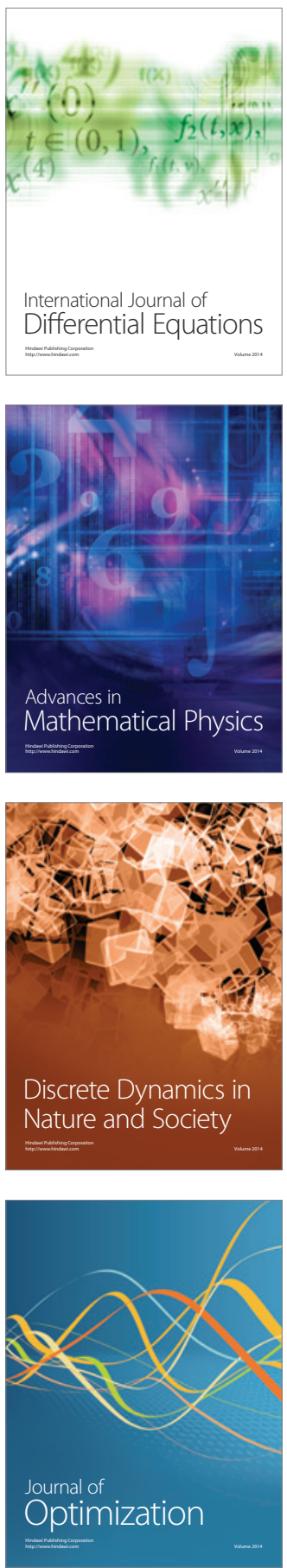